\title{
Novel ABCD Formula to Diagnose and Feature Ranking of Melanoma
}

\author{
Reshma. M \\ Research Scholar at Sathyabama Institute of Science and \\ Technology, Dept. of E \& C \\ UBDT College of Engineering Davangere, India
}

\author{
B. Priestly Shan \\ Principal, \\ Eranad Knowledge City-Technical Campus \\ Manjeri, Kerala
}

\begin{abstract}
A prototype of skin cancer detection system for melanoma diagnoses in early stages is very important. In this paper, a novel technique is proposed for Skin malignant growth identification based on feature parameters, color shading histogram, to improve the diagnosis method by optimizing the ABCD formula. Features are extracted like Shape, Statistical, GLCM texture, Color, Wavelet transform, Texture. Once the features are extracted we found the most prominent features by assigning a rank. We have calculated parameters such as sensitivity, specificity, accuracy for checking the imperceptibility and robustness of the proposed approach. Also, Correlation analysis is made between traditional and proposed TDS equation using Karl Pearson's method.
\end{abstract}

Keywords-Karl Pearson's method; gray level co-occurrence matrix (GLCM); wavelet transform; melanoma; dermoscopy

\section{INTRODUCTION}

Cancer is an anomalous cell development. In contrast to typical cells, malignant cells are grown to develop and shape in to new strange cells. In the same way, skin cancer is an anomalous development of melanocytic cells in the skin. These melanocytic cells produce melanin, when it is exposed to harmful radiations, the external parameters like Ultraviolet radiations and hereditary variables will cause harm to DNA cells which in turn results in the creation of strange melanocytic cells and they group together to shape harmful development. Because of the malignant growth of the melanocytic cells, skin cancer is also called Melanoma. The early stage of melanoma is very hard to recognize based on the grounds of threatening melanoma which can impart numerous clinical highlights to an atypical nevus. A few examinations have portrayed symptomatic precision rates extending from 50-75\%, demonstrating a requirement for extra indicative apparatuses. The presentation of Dermoscopy, named as Epiluminescence microscopy (ELM), has opened another measurement in the examination of pigmented skin sores and particularly, in the distinguishing proof of the early period of cutaneous harmful melanoma [1], [2]. Despite the fact that Dermoscopy enhances the analytic precision for melanoma, it cannot supplant histopathology examination. Some lesions, especially early melanomas, may lack specific Dermoscopy features and are difficult to diagnose.

This paper includes images of melanomas with multiple characteristic features to identify the cause for melanoma. Owing to their characteristic, some of them are relatively featureless. The first part of the proposed system is to recognize the image whether it is benign or malignant melanoma. Developed a New TDS Equation and correlated the Existed TDS with New TDS. In the second part, ranking the prominent features of melanoma images are evaluated with the proposed ranking system. Melanoma is spreading in people of the worldwide which remains practically incurable. Hence it is necessary to have early detection of this disease to reduce the death rate. Identifying melanomas is not an easy task and despite of this known fact, there are some exclusive standards for a system Dermoscopy or ELM the assessment of pigmented skin sores with this strategy is regularly greatly subjective and complex. With the end goal to conquer the issue of subjective elucidation, techniques dependent on the numerical investigation of pigmented skin injuries such as digital Dermoscopy analysis, have been recently been developed [3]. The traditional diagnosis, dermatoscopy and interpretation evaluate forms, colors, dimensions patterns and textures. The $\mathrm{ABCD}$ rule, also referred to as the STOLZ method developed by Stolz et al., used to differentiate benign melanocytic lesions with melanoma by Dermoscopy. Objective mathematical definition evaluations offer stable and reproducible measurements. The length of time required for every examination is the great limitation of subjective algorithms. Subjective algorithms have been widely demonstrated to be inefficient and inconsistent with the common sense and experience of experts. To achieve an effective way of early detection of skin cancer without unnecessary skin biopsies, digital images of melanoma skin lesions have been investigated. The main objective of the diagnosis is to develop software to help clinicians practice daily. The system therefore needs to be easy to use, fast and not based on subjective assessment. As many features exist in order to identify melanoma but the main objective of the proposed system is to select the best features to identify melanoma which may be done by assigning the ranks to the extracted features. Extracted 40 attributes from a set of 6 main features where these features are trained with a linear discriminant classifier and ranked the features with highest priority mentioned in Part B.

\section{Problem Statement}

Over the past decade, epidemiologists have expressed concern that screening could lead to the excision of inconsequential cancer, i.e. melanomas that would never have become life- threatening tumors, a phenomenon referred to by the misleading term "over diagnosis". Without any strong 
evidence, speculation has been accepted throughout the world and incipient melanomas have been trivialized. Quick analysis and diagnoses are necessary when patients are in confusion state. Too many irrelevant features will not only complicate the classifier, but also reduce the accuracy of the classification. The size of the tumors is very small at an early stage, so that prediction is ambiguous. Many times, even with the good image, patients are made to wait for the doctor's appointment to diagnose, this delay which can also pose a threat to the critical condition of the patient. Some may be wrong because of human error prediction. A new method is therefore used to demonstrate the success of melanoma detection and superiority among the characteristics without any human assistance to ensure good efficiency, accurate results without biopsy. So our aim is to design, implement and test an automated knowledge-based clinical decision support system for melanoma detection and grading using soft computing techniques.

\section{PROPOSED BLOCK DIAGRAM}

Fig. 1 represents a block diagram for melanoma identification using TDS and Features ranking based LDA classifier. The sources of images in this paper are from the ISIC-Archive and $\mathrm{PH} 2$ dataset [4]. Part A briefs Preprocessing, segmentation, Feature Extraction, melanoma detection using Proposed ABCD (TDS) Formula, Comparing the traditional TDS equation with Proposed TDS.

\section{A. Melanoma Identification}

For Preprocessing the digital image is given as system input. Most Dermoscopy images may contain certain unwanted particles such as thin and thick hair, air bubbles, gel and illumination effects. Therefore, robust ways to remove noise and unwanted particles have emerged. Some of these particles, like air bubble or oil, become less common when Dermoscopy are developed. The next stage of the processing involves de-noising. Median Filter is used to detect the noises present in the image. This filter identifies and eliminates the unwanted. Median filter obtains a higher PSNR value and restores the image with better image quality compared to other filters. The filtered RGB image is converted to Gray Image via Principal Component Analysis Which effectively preserves color and texture discrimination. Followed by a DWT2 for image enhancement, to obtain a multi-scale representation of the original and offer a good representation of the highfrequency components (edges) that allow the image to be represented more compactly. The Image is then subjected to segmentation. Segmentation separates suspected lesion from ordinary skin. The Sobel operator precedes the borders in which the gradient is better [5], [6]. Asymmetry, Border Irregularity, variation in pigment color, and Dimensionality [7] are the features extracted to impose whether Traditional (original) TDS or new TDS enhances determination of melanoma. For Asymmetry (A) calculation, program gathers the border and centroid estimation of an Image. We circularly move the qualities in the range $\mathrm{A}$ by $\mathrm{K}$ to the measurement and afterward partition the picture into two sections (A and $\mathrm{B}$ ) once by vertical line and again by even line. In the two cases, vertical and even asymmetry is resolved. To check the symmetry factor, discover the similitude between the left and right parts. Outwardly, on the off chance we pivot the left piece of the sore with 180 degrees around the vertical line and cover the left part on the right, it is possible that they are almost indistinguishable or there are diverse parts between them. Asymmetry Index (AI) will be equivalent to average of vertical and even asymmetry as follows:

$$
A I=\frac{\left(A I_{1}+A I_{2}\right)}{2}
$$

The score of AI is $0,1,2$ when the symmetry of the lesion is in both directions, one direction, asymmetry in both directions respectively. Compactness index(CI), fractal dimension, Abruptness of edge, mean and variance of pigment transition [8] are the attributes required to define for Border Irregularity of lesion. The density index (Compactness Index) is the measurement of the most popular barrier form estimated unanimously by 2D objects. Fractal measurement has qualities of self-comparability and properties of scale/estimate. Each area has a fractal which is of an alternate scale with the whole fractal. This component makes for fractal pressure systems. Measurement estimate is generally a number, as the line has measurement 1 , the field has measurement 2 , and the solid shape has three measurements, etc. The fractal measurement is, be that as it may, an unusual viewpoint that could be worth portions. This fractal measurement can be utilized as a picture trademark. Fractal measurements can be determined utilizing the case figuring strategy. This technique partitions the picture into boxes in various sizes (p). Utilize the crate framework, which partitions the picture into the containers in shifting sizes. $N(p)$ is surveyed as the quantity of pixels containing parts of the damage hindrance. There are distinctive pixel sizes and $\mathrm{p}$ as incline fd relapse line $\log (\mathrm{p})$ vs $\log (\mathrm{N}(\mathrm{p}))$.

$$
N(p)=\lambda p-f d
$$

Above condition was reached out to

$\log \left[\frac{1}{N(p)}\right]=f d * x * \log (p)-\log (\lambda)$

The component of Hausdorff is a proportion of harshness and fills in as a proportion of the nearby size of a space, considering the separation between its points. Compactness Index (CI) can be determined utilizing the underneath condition,

$$
C I=\frac{(P * P)}{(4 \pi A)}
$$




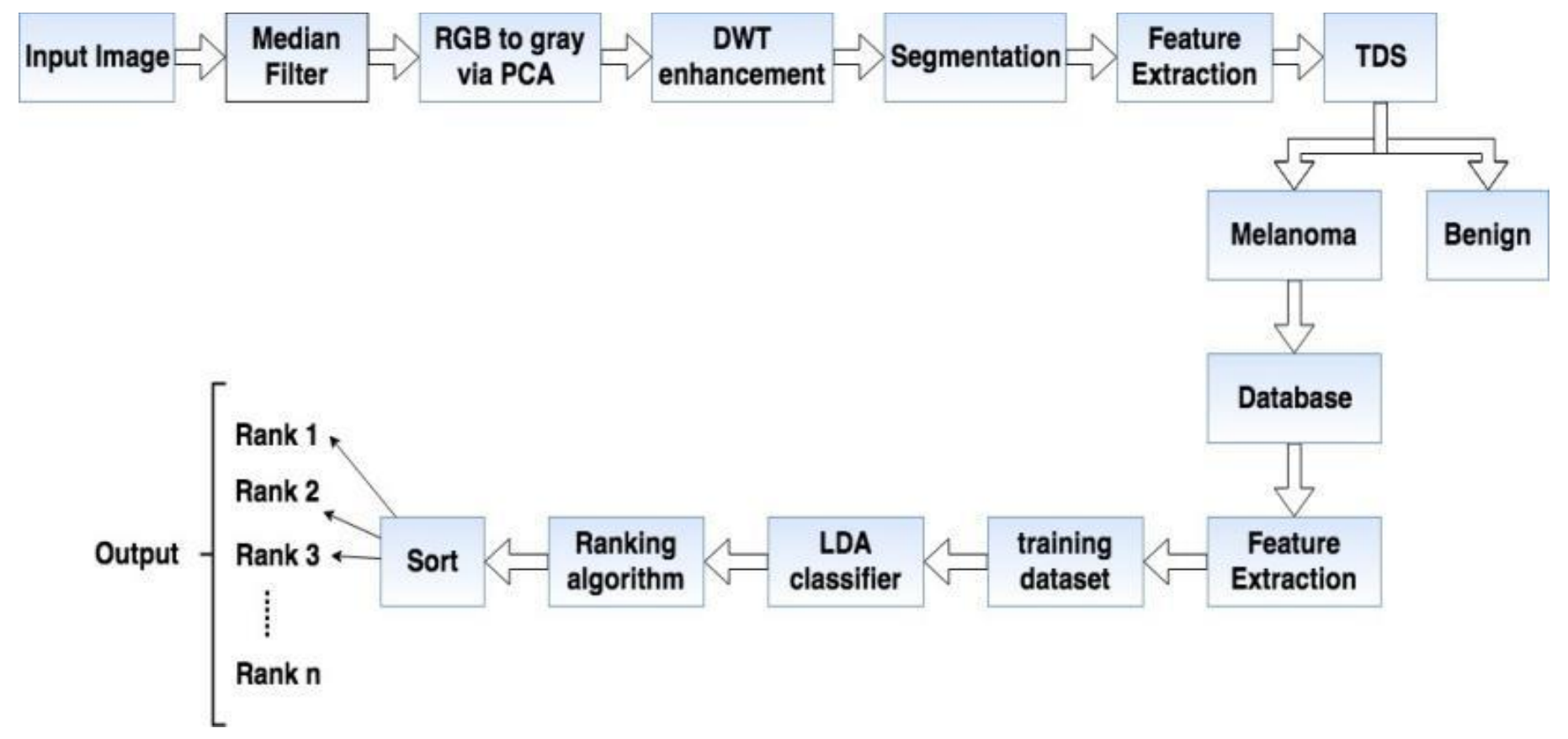

Fig. 1. Melanoma Identification using TDS and Features Ranking based LDA Classifier.

Where, $\mathrm{P}$ is the perimeter and $\mathrm{A}$ is the area of the lesion. This essential element clarifies change of skin pigmentation between the sore and encompassing skin. Sharp edge is steep perilous when blurring gradually, does not demonstrate an unsafe sore. For that, we consider part previously $(i, j)$ of the first shading picture as the main three components are weighted a similar shading. At that point we gauge the inclination greatness of force segment luminance (lum) along the limit prior to $\mathrm{C}$ of the skin injury. Acquired a set of angle greatness estimation of $N$, e $(n)(1 \leq n \leq N$, where $N$ is the constraining example estimate) that depicts locally the progress between the damage and setting purposes of skin on each side. To portray more globally, utilized the mean and variance of the gradient magnitude values e (n) which portrays the dimension of steepness and generic variations. Equations are listed below for luminance, mean and variance of the gradient.

$\operatorname{lum}(i, j)=\frac{r(i, j)+g(i, j)+b(i, j)}{3}$

$M_{e}=\frac{1}{n} \sum_{n=1}^{n} e(n)$

$V_{e}=\frac{1}{n} \sum_{n=1}^{n} e^{2}(n)-m_{e}^{2}$

For Edge Abruptness sore with sporadic points of confinement (sudden edge) has a critical contrast in outspread separation. Eliminate the estimation of abnormalities by dissecting the distribution of the radial distance difference.

$C_{r}=\frac{\frac{1}{p_{L}} \sum P \varepsilon C\left(d_{2}\left(P * G_{L}\right)-m_{d}\right)^{2}}{m_{d}^{2}}$

Where $m_{d}$ is the mean distance of $d_{2}$ between the point barrier centered and the $G_{L}$. The score of $B$ is 0 to 8 . An early indication of melanoma is the improvement of sore shading varieties. Since melanoma cells develop in the shade of the producer, they are frequently brilliant around dark colored or dark, contingent upon the generation of the melanin color in the skin at various depths. We have made a shading histogram for dark, white, dark, dim, darker, yellow, green, barrel shaped, fuchsia hues. The entire picture is examined and the quantity of pixels having a place with every district is checked. The shade color is one of the lesion hues when the quantity of pixels surpasses 0.1 percent of the aggregate number of pixels in the sore picture. The normal sore distance across can be resolved with the diameter equation.

$D=\sqrt{\frac{4 A}{\pi}}$

On the off chance that $\mathrm{A}$ is the area of the lesion, the measurement for a favorable sore ought to be under $6 \mathrm{~mm}$. Dangerous development in width in excess of $6 \mathrm{~mm}$. In Stolz algorithm[9] presented the ABCD rule and utilized by dermatologists to evaluate the danger of threat of a pigmented sore in the recognition of skin injuries. Notwithstanding its estimation speed, this empowers an increasingly objective and regenerative conclusion of skin malignancies. The traditional formula is used to determine the melanoma by using the equation.

$T D S=[(A * 1.3)+(B * 0.1)+(C * 0.5)+(D * 0.5)]$

The end result is 1.00 to 4.75 as benign, 4.775 to 5.45 as suspicious, more than 5.45 as malignant melanoma. A new TDS equation is formulated to diagnose melanoma which is given below in equation (11). We assumed that the ABCD formula for computing a new TDS must be additionally a linear mixture of nine attributes [10], [11], [12]. The coefficients or weighting factor of new developed TDS are listed below in Table 1. 
TABLE I. EXPLORED COEFFICIENT RANGES FOR NINE ATTRIBUTES FROM THE MELANOMA DATA SET

\begin{tabular}{|l|l|}
\hline Attribute Tested & Weighting Factor \\
\hline Asymmetry Index & 1.3 \\
\hline Border & 0.1 \\
\hline Color Black & 0.5 \\
\hline Color white & 0.5 \\
\hline Color Red & 0.5 \\
\hline Color blue & 0.5 \\
\hline Color DarkBrown & 0.3 \\
\hline Color lightbrown & 0.4 \\
\hline Diameter & 0.5 \\
\hline
\end{tabular}

New TDS $=c 1 *$ AsymmetryIndex $+\mathrm{c} 2 *$ Border irregulari ty +

$\mathrm{c} 3 *$ Color Black $+\mathrm{c} 4 *$ Color White $+\mathrm{c} 5 *$ Color Red +

$c 6 *$ Color Blue $+\mathrm{c} 7 *$ Color DarkBrown +

c $8 *$ Color Lightbrown $+c 9 *$ Diameter

On substituting the values of coefficients, the New TDS equation is obtained as

New TDS $=1.3 *$ Asy mmetryIndex $+0.1 *$ Border irregulari ty

$+0.5 *$ Color Black $+0.5 *$ Color White

$+0.5 *$ Color Red $+0.5 *$ Color Blue

$+0.3 *$ Color DarkBrown $+0.4 *$ Color

Lightbrown $+0.5 *$ Diameter

\section{B. Ranking the Features of Melanoma}

In this part features of melanoma like Shape Features, Statistical feature , GLCM texture feature, Color feature [13], Wavelet transform and Texture feature [14], [15] are extracted from Images. A set of attributes were extracted from these 6 features. Area, length of the major axis, length of the small axis, centroid position and perimeter are the 5 attributes obtained from shape based characteristics. The outcome is a vector of shape highlights with five elements obtained by utilizing mat lab regionprops for segmented binary Image .Statistical features attributes are entropy, mean, standard deviation, median, skewness, and kurtosis. The output is a collection of 6 attributes from the class of Statistical features. Auto Correlation, cluster Prominence, cluster Shade, contrast, correlation, difference Entropy ,difference Variance, dissimilarity, energy, entropy, homogeneity, information Measure of Correlation 1, information Measure Of Correlation 2, inverse Difference, maximum Probability, Average, sum of Entropy, sum Of Squares Variance, Variance are the 19 elements calculated for GLCM features. The color characteristics include mean, normal derivation. These attributed are extracted with the descriptor Color Moment (CM). The common moments are mean, Standard deviation can be calculated as follows, respectively.

$\mu_{i}=\frac{1}{N} \sum_{J=1}^{N} f_{i J}$
$\sigma_{i}=\sqrt{\frac{1}{N} \sum_{J=1}^{i}\left(f_{i J}-\mu_{i}\right)^{2}}$

The mean and Standard Deviation for red, green, blue components present in the image are calculated. So a set of 6 values is obtained from color feature. For wavelet transform feature, we are using dwt2 type transform to extract 2 elements mean Coefficients, standard Coefficient features. Mean Amplitude, Mean-squared Energy are 2 attributes extracted from Texture Feature. Thus a set 40 attributes are extracted from six main features. These attributes are used as training data. LDA classifier [16] is applied on each feature resulting a vector value [17]. Classified feature output are collected and fed to the Ranking Algorithm. Rank Algorithm ranks the features in $\mathrm{X}$ using an independent binary classification evaluation criterion, by default a two-sample Ttest. $\mathrm{X}$ is a matrix in which each column is a vector observed and the number of rows corresponds to the original number of characteristics. It uses correlation information to out weight the $\mathrm{Z}$ value of potential characteristics using the equation.

$X=Z *(1-A L P H A *(R H O))$

Where RHO is the average cross correlation coefficient of absolute values between the test feature and all previously selected features. Rank features applies independent normalization across the observations for every feature. Sort the vector values in decreasing order, the highest value assigns a rank 1 .

\section{RESULTS AND DISCUSSION}

In order to test the Part A performance of the proposed diagnostic method, we used 82 images with 78 cancer images and 4 non - cancer images. The figures of input image, Restored image, DWT2 output image, idwt image, edge detected image, segmented skin lesion, segmented image are listed below in Fig. 2 to 4 shows a GUI for the image detected as Benign and Melanoma Fig. 5 and 6 shows a Polynomial curve fitting for Hausdorff dimension for benign and melanoma image. Used New TDS or proposed TDS to distinguish the image as melanoma or nonmelanoma. A Pearson Correlation [18] of 0.88 is achieved between the Traditional TDS and Proposed TDS which is shown in Fig. 7. The Pearson correlation coefficient, often referred to as the Pearson $\mathrm{R}$ test, is a statistical formula that measures the strength between variables and relationships [19]. The qualities acquired by experimentation for Sensitivity and Specificity are 0.96 and 0.75 separately shown in Table 3. Authors found that the coordinating project framework performed $85.5 \%$ in recognizing the Melanoma skin disease. The Accuracy calculated from sensitivity and specificity is about $95 \%$ which is improved compared to earlier techniques. The results of part 2 shows shape features as the most prominent feature for early diagnosis of melanoma. Fig. 8 shows Ranks ordered to the features. Rank 2 is Statistical feature, color feature is in Rank 3, wavelet feature is in fifth position, and last Rank is Gabor feature. Table 2 shows comparison between investigation and system results for proposed TDS. 


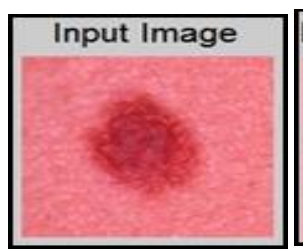

(a)

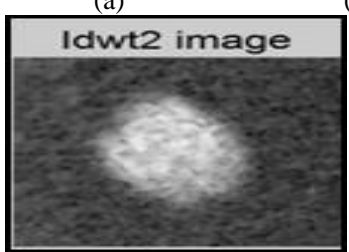

(d)

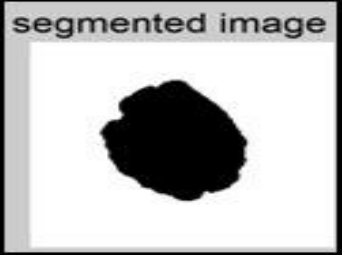

(f)

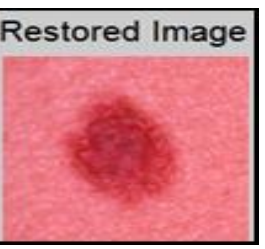

(b)

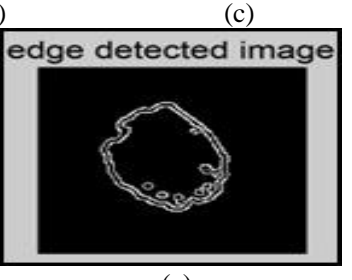

(e)

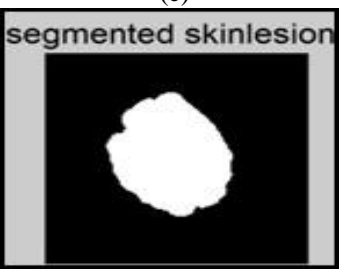

(g)

in

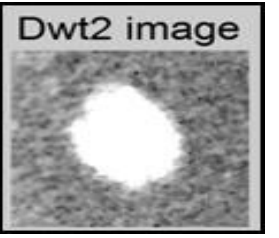

Fig. 2. Input Image (b) Restored Image (c) DWT2 Output Image (d) Idwt Image (e) Edge Detected Image (f) Segmented Skin Lesion (g) Segmented

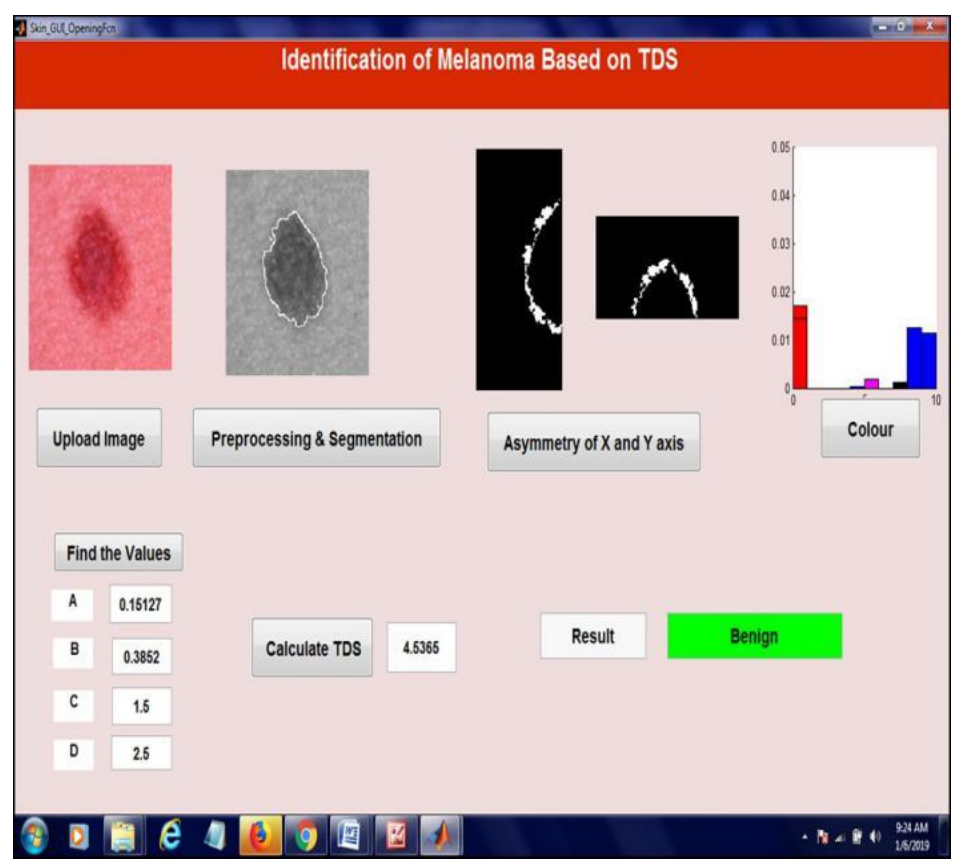

Fig. 3. Identified as Benign. Image.

TABLE II. COMPARISONS BETWEEN EXAMINATION AND FRAMEWORK RESULTS FOR PROPOSED TDS

\begin{tabular}{|c|c|c|c|c|c|c|c|c|c|c|c|c|}
\hline Images & A & B & Black & White & Red & Blue & D brown & L brown & D & $\begin{array}{l}\text { TDS } \\
\text { (Proposed) }\end{array}$ & Conclusion & Result \\
\hline M004 & 0.0576 & 0.4666 & 0.0 & 0.0003 & 0.0 & 0.0079 & 0.0076 & 0.0067 & 7.0864 & 7.6312 & Melanoma & True \\
\hline M013 & 0.2816 & 0.3991 & 0.001 & 0.0012 & 0.0 & 0.0007 & 0.0144 & 0.0143 & 4.7844 & 5.4837 & Melanoma & True \\
\hline M022 & 0.1751 & 0.646 & 0 & 0 & 0.0 & 0.001 & 0.0097 & 0.0096 & 5.6428 & 6.4758 & Melanoma & True \\
\hline M026 & 0.348 & 0.4512 & 0 & 0 & 0.0 & 0.0005 & 0.0037 & 0.0036 & 4.9293 & 5.7337 & Melanoma & True \\
\hline M029 & 0.1284 & 0.3995 & 0.0 & 0.0012 & 0.0 & 0.0001 & 0.0216 & 0.0215 & 6.0438 & 6.5987 & Melanoma & True \\
\hline M035 & 0.0839 & 0.5026 & 0.0 & 0 & 0.0 & 0.019 & 0.0019 & 0.0019 & 7.2379 & 7.8369 & Melanoma & True \\
\hline M040 & 0.2371 & 0.4015 & 0 & 0 & 0.0 & 0.0091 & 0.0015 & 0.0015 & 5.4589 & 6.1039 & Melanoma & True \\
\hline M043 & 0.1595 & 1.0216 & 0.0 & 0.0014 & 0.0 & 0.0103 & 0.0276 & 0.0276 & 8.2824 & 9.5027 & Melanoma & True \\
\hline M046 & 0.1595 & 0.5238 & 0 & 0 & 0.0 & 0.0117 & 0.0012 & 0.0012 & 8.0492 & 8.74 & Melanoma & True \\
\hline M049 & 0.483 & 0.8652 & 0.0 & 0 & 0.0 & 0.0008 & 0.0135 & 0.0134 & 6.9564 & 8.3217 & Melanoma & True \\
\hline M054 & 0.2217 & 0.3791 & 0 & 0 & 0.0 & 0.0006 & 0.0074 & 0.0074 & 5.6935 & 6.3037 & Melanoma & True \\
\hline M056 & 0.2551 & 0.5479 & 0 & 0 & 0.0 & 0.0005 & 0.0071 & 0.007 & 7.0803 & 7.8921 & Melanoma & True \\
\hline M070 & 0.2176 & 0.5946 & 0.0016 & 0 & 0.0 & 0.0056 & 0.0064 & 0.0064 & 6.6626 & 7.4859 & Melanoma & True \\
\hline M139 & 0.1308 & 0.4016 & 0 & 0 & 0.018 & 0 & 0.0132 & 0.013 & 5.9652 & 6.5158 & Melanoma & True \\
\hline
\end{tabular}




\begin{tabular}{|c|c|c|c|c|c|c|c|c|c|c|c|c|}
\hline M141 & 0.3155 & 0.386 & 0.0009 & 0 & 0.011 & 0 & 0.0117 & 0.0116 & 6.9415 & 7.6573 & Melanoma & True \\
\hline M142 & 0.0749 & 0.4208 & 0.0007 & 0 & 0.036 & 0.0006 & 0.0354 & 0.0352 & 6.3813 & 6.9204 & Melanoma & True \\
\hline M143 & 0.2578 & 0.4502 & 0.0003 & 0 & 0.012 & 0 & 0.017 & 0.017 & 6.7777 & 7.504 & Melanoma & True \\
\hline M145 & 0.0827 & 0.3478 & 0 & 0 & 0.016 & 0 & 0.0159 & 0.0159 & 7.7664 & 8.216 & Melanoma & True \\
\hline M146 & 0.1004 & 0.3901 & 0.0008 & 0.0008 & 0.04 & 0.0011 & 0.0276 & 0.0265 & 7.5802 & 8.1108 & Melanoma & True \\
\hline M147 & 0.1397 & 0.8486 & 0.0002 & 0.0001 & 0.04 & 0.0003 & 0.03 & 0.0287 & 5.2704 & 6.2995 & Melanoma & True \\
\hline M148 & 0.3347 & 1.0818 & 0.0013 & 0 & 0.0 & 0.0004 & 0.0064 & 0.0064 & 5.6942 & 7.1209 & Melanoma & True \\
\hline M149 & 0.1571 & 0.6063 & 0 & 0 & 0.021 & 0 & 0.0177 & 0.0176 & 7.7029 & 8.4889 & Melanoma & True \\
\hline M150 & 0.1247 & 0.4035 & 0 & 0 & 0.022 & 0 & 0.0218 & 0.0218 & 6.0661 & 6.6205 & Melanoma & True \\
\hline M152 & 0.1101 & 0.3568 & 0.0007 & 0 & 0.011 & 0.0005 & 0.0159 & 0.0159 & 5.6755 & 6.1596 & Melanoma & True \\
\hline M153 & 0.197 & 0.3934 & 0 & 0.0001 & 0.0 & 0 & 0.0113 & 0.0113 & 7.0782 & 7.6791 & Melanoma & True \\
\hline M155 & 0.1146 & 0.4404 & 0.002 & 0.0007 & 0.033 & 0.0002 & 0.0356 & 0.0356 & 5.4671 & 6.0648 & Melanoma & True \\
\hline M156 & 0.4295 & 0.4258 & 0.0001 & 0 & 0.03 & 0 & 0.0313 & 0.0313 & 5.2392 & 6.1316 & Melanoma & True \\
\hline M157 & 0.1422 & 0.4079 & 0.0002 & 0 & 0.021 & 0 & 0.0202 & 0.0201 & 6.3621 & 6.9367 & Melanoma & True \\
\hline M159 & 0.2306 & 0.5747 & 0 & 0.0002 & 0.03 & 0 & 0.0242 & 0.0236 & 6.53 & 7.367 & Melanoma & True \\
\hline M161 & 0.2617 & 0.6556 & 0.0016 & 0.0001 & 0.031 & 0 & 0.0298 & 0.0296 & 4.9741 & 5.9284 & Melanoma & True \\
\hline M162 & 0.1709 & 0.4206 & 0 & 0.0002 & 0.017 & 0 & 0.0144 & 0.0142 & 5.0048 & 5.6148 & Melanoma & True \\
\hline M163 & 0.5192 & 0.5273 & 0.0005 & 0 & 0.025 & 0 & 0.0235 & 0.0234 & 3.1784 & 4.2541 & Benign & False \\
\hline M164 & 0.2795 & 0.7271 & 0.0004 & 0 & 0.032 & 0 & 0.03 & 0.0299 & 4.5293 & 5.5729 & Melanoma & True \\
\hline M165 & 0.3704 & 0.8327 & 0.0003 & 0 & 0.02 & 0 & 0.0186 & 0.0184 & 3.5568 & 4.7828 & Benign & False \\
\hline M166 & 0.1502 & 0.5246 & 0.0001 & 0.0006 & 0.025 & 0 & 0.0219 & 0.0218 & 5.495 & 6.198 & Melanoma & True \\
\hline M168 & 0.2619 & 0.7725 & 0 & 0 & 0.022 & 0 & 0.0223 & 0.0222 & 5.1171 & 6.1783 & Melanoma & True \\
\hline M171 & 0.1524 & 0.8182 & 0 & 0.0005 & 0.031 & 0.0022 & 0.0247 & 0.0244 & 4.7786 & 5.7831 & Melanoma & True \\
\hline M172 & 0.3027 & 0.5046 & 0.0002 & 0 & 0.02 & 0 & 0.0196 & 0.0196 & 4.3943 & 5.2252 & Benign & False \\
\hline M173 & 0.2935 & 0.7068 & 0.0008 & 0.0006 & 0.036 & 0 & 0.0377 & 0.0373 & 4.4165 & 5.4616 & Melanoma & True \\
\hline M174 & 0.3362 & 0.4706 & 0 & 0.0001 & 0.032 & 0.0001 & 0.0249 & 0.0246 & 5.4738 & 6.3138 & Melanoma & True \\
\hline M175 & 0.1856 & 0.5307 & 0 & 0.0005 & 0.019 & 0.0001 & 0.0183 & 0.0183 & 5.703 & 6.4418 & Melanoma & True \\
\hline M278 & 0.2724 & 0.9678 & 0 & 0 & 0.015 & 0 & 0.0149 & 0.0149 & 5.5179 & 6.776 & Melanoma & True \\
\hline
\end{tabular}




\begin{tabular}{|c|c|c|c|c|c|c|c|c|c|c|c|c|}
\hline M279 & 0.2182 & 0.6015 & 0 & 0 & 0.021 & 0 & 0.02 & 0.0199 & 5.9508 & 6.7949 & Melanoma & True \\
\hline M285 & 0.3139 & 1.3484 & 0 & 0 & 0.017 & 0 & 0.017 & 0.017 & 4.3369 & 6.0197 & Melanoma & True \\
\hline M288 & 0.3541 & 0.7634 & 0 & 0.0011 & 0.042 & 0 & 0.0426 & 0.0424 & 5.9523 & 7.1212 & Melanoma & True \\
\hline M289 & 0.2132 & 0.8163 & 0.0007 & 0 & 0.045 & 0 & 0.0448 & 0.0448 & 6.4397 & 7.5233 & Melanoma & True \\
\hline M291 & 0.276 & 0.7973 & 0 & 0 & 0.015 & 0 & 0.0155 & 0.0155 & 4.3996 & 5.4915 & Melanoma & True \\
\hline M292 & 0.1858 & 0.7717 & 0 & 0 & 0.022 & 0 & 0.0218 & 0.0217 & 5.3879 & 6.3715 & Melanoma & True \\
\hline M293 & 0.168 & 0.4095 & 0.0012 & 0.0003 & 0.04 & 0 & 0.0365 & 0.0363 & 7.4067 & 8.0306 & Melanoma & True \\
\hline M294 & 0.1782 & 0.5807 & 0 & 0 & 0.014 & 0 & 0.0143 & 0.0141 & 6.071 & 6.847 & Melanoma & True \\
\hline M299 & 0.3776 & 0.8753 & 0.0 & 0 & 0.0 & 0.0006 & 0.0294 & 0.0289 & 5.6134 & 6.9041 & Melanoma & True \\
\hline M301 & 0.0602 & 0.4354 & 0.0 & 0 & 0.0 & 0 & 0.0255 & 0.0254 & 5.0729 & 5.5999 & Melanoma & True \\
\hline M302 & 0.2687 & 0.6532 & 0.0 & 0 & 0.0 & 0 & 0.034 & 0.0338 & 5.0763 & 6.0395 & Melanoma & True \\
\hline M307 & 0.5445 & 0.7881 & 0.0 & 0 & 0.0 & 0 & 0.0345 & 0.034 & 4.3063 & 5.6843 & Melanoma & True \\
\hline M314 & 0.0681 & 0.395 & 0.0 & 0.0002 & 0.0 & 0 & 0.0292 & 0.0291 & 5.1226 & 5.6218 & Melanoma & True \\
\hline M390 & 0.2021 & 0.5852 & 0.0 & 0 & 0.02 & 0.0003 & 0.0181 & 0.0179 & 4.9995 & 5.8103 & Melanoma & True \\
\hline M395 & 0.6173 & 1.1877 & 0.0 & 0 & 0.0 & 0.0026 & 0.0132 & 0.0128 & 5.1735 & 6.9982 & Melanoma & True \\
\hline M398 & 0.2365 & 0.6024 & 0.0 & 0 & 0.0 & 0 & 0.0169 & 0.0169 & 5.9228 & 6.7819 & Melanoma & True \\
\hline M404 & 0.1736 & 0.4952 & 0 & 0 & 0.0 & 0 & 0.0158 & 0.0159 & 4.7957 & 5.4813 & Melanoma & True \\
\hline M414 & 0.4505 & 0.7971 & 0 & 0 & 0.0 & 0.0032 & 0.0137 & 0.0134 & 4.4982 & 5.7666 & Melanoma & True \\
\hline M463 & 0.1499 & 0.4967 & 0.0 & 0.0001 & 0.0 & 0 & 0.0132 & 0.0132 & 5.0318 & 5.6947 & Melanoma & True \\
\hline M466 & 0.4996 & 0.4841 & 0 & 0 & 0.0 & 0 & 0.0072 & 0.0072 & 4.9446 & 5.9364 & Melanoma & True \\
\hline M469 & 0.334 & 0.4169 & 0 & 0 & 0.0 & 0 & 0.0097 & 0.0097 & 4.8251 & 5.5877 & Melanoma & True \\
\hline M482 & 0.1823 & 0.3382 & 0.0 & 0 & 0.0 & 0.0002 & 0.0091 & 0.0091 & 5.246 & 5.7779 & Melanoma & True \\
\hline M484 & 0.3068 & 0.6788 & 0 & 0 & 0.0 & 0 & 0.0081 & 0.0081 & 6.6756 & 7.671 & Melanoma & True \\
\hline M487 & 0.2678 & 0.4784 & 0 & 0 & 0.0 & 0 & 0.0081 & 0.0081 & 7.1008 & 7.8567 & Melanoma & True \\
\hline M502 & 0.3336 & 0.6567 & 0 & 0 & 0.0 & 0 & 0.006 & 0.006 & 5.4028 & 6.4001 & Melanoma & True \\
\hline M511 & 0.3661 & 0.51 & 0 & 0 & 0.0 & 0 & 0.0134 & 0.0133 & 6.3882 & 7.2808 & Melanoma & True \\
\hline M513 & 0.1351 & 0.3762 & 0 & 0 & 0.01 & 0 & 0.0101 & 0.01 & 5.6447 & 6.1682 & Melanoma & True \\
\hline M517 & 0.2304 & 0.672 & 0 & 0 & 0.0 & 0 & 0.0072 & 0.0072 & 5.2702 & 6.1812 & Melanoma & True \\
\hline
\end{tabular}




\begin{tabular}{|c|c|c|c|c|c|c|c|c|c|c|c|c|}
\hline M521 & 0.082 & 0.3866 & 0 & 0 & 0.0 & 0.0001 & 0.016 & 0.0159 & 6.1319 & 6.62 & Melanoma & True \\
\hline M522 & 0.3688 & 0.6476 & 0 & 0 & 0.0 & 0 & 0.0086 & 0.0086 & 4.8355 & 5.8621 & Melanoma & True \\
\hline M531 & 0.1958 & 0.3586 & 0 & 0 & 0.0 & 0 & 0.0102 & 0.0102 & 5.4047 & 5.9706 & Melanoma & True \\
\hline M533 & 0.3066 & 0.5325 & 0 & 0 & 0.0 & 0 & 0.0136 & 0.0136 & 5.2209 & 6.0762 & Melanoma & True \\
\hline M548 & 0.2758 & 0.4173 & 0 & 0 & 0.0 & 0 & 0.0054 & 0.0054 & 5.4162 & 6.1159 & Melanoma & True \\
\hline M549 & 0.2918 & 0.7369 & 0 & 0 & 0.0 & 0 & 0.0168 & 0.0168 & 5.8533 & 6.9024 & Melanoma & True \\
\hline NM146 & 0.0012 & 0.2141 & 0 & 0 & 0 & 0 & 0.0 & 0.0071 & 1.3451 & 1.5633 & Benign & True \\
\hline NM 553 & 0.1512 & 0.2171 & 0 & 0 & 0 & 0 & 0.0012 & 0.0101 & 2.1812 & 2.5539 & Benign & True \\
\hline NM 554 & 0.2318 & 0.3275 & 0 & 0.0032 & 0 & 0 & 0.0043 & 0.0084 & 2.0012 & 2.5668 & Benign & True \\
\hline
\end{tabular}

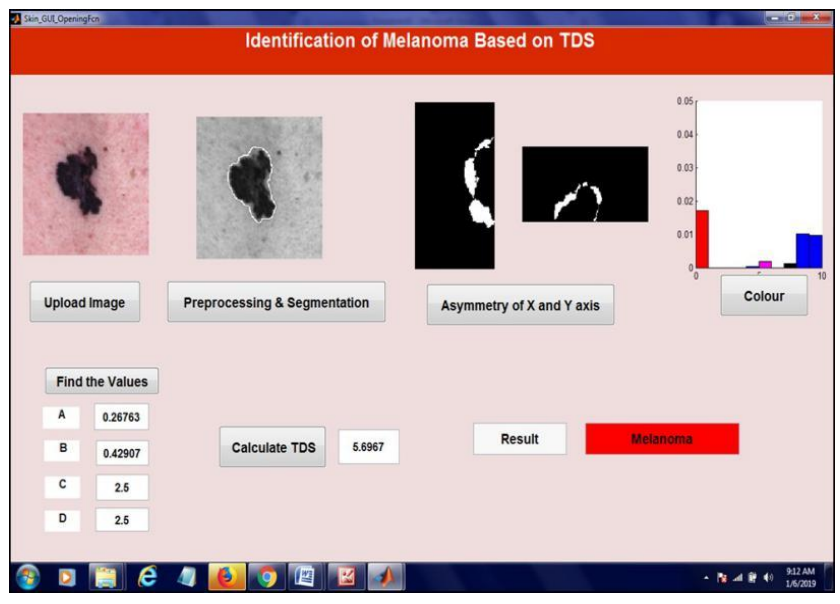

Fig. 4. Identified as Melanoma.

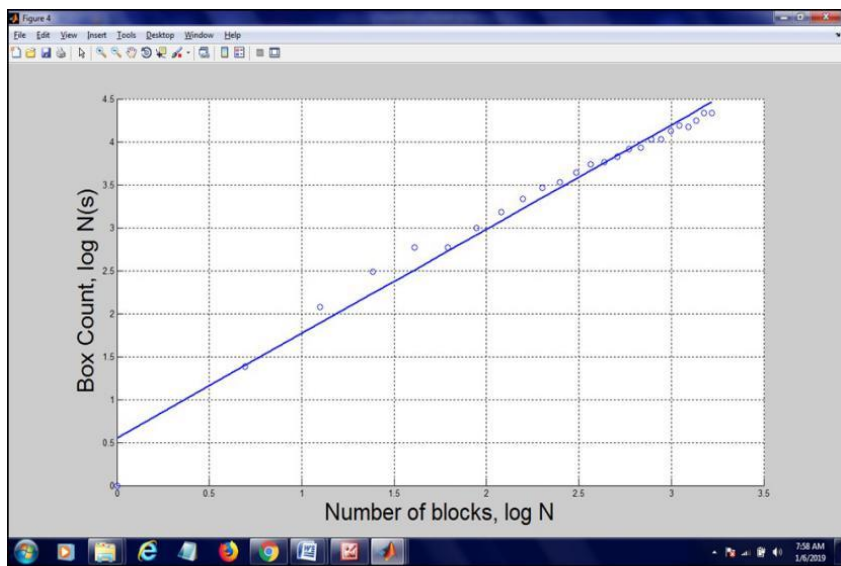

Fig. 5. Polynomial Curve Fitting for Hausdorff Dimension for benign Image.

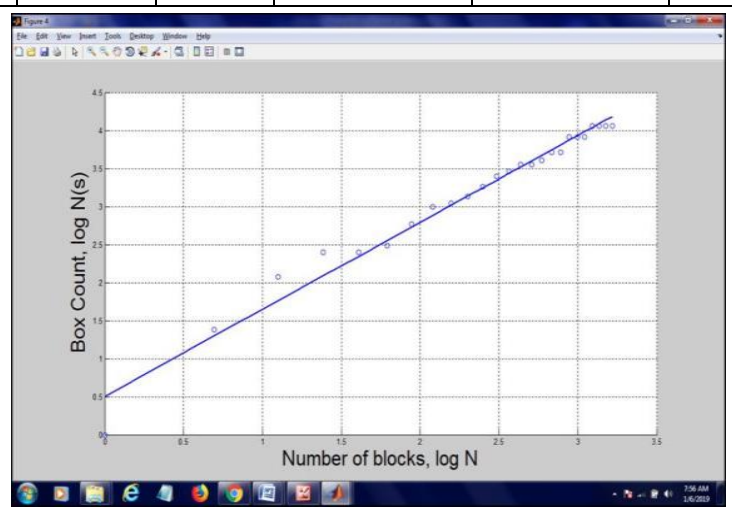

Fig. 6. Polynomial Curve Fitting for Hausdorff Dimension for Melanoma Image.

\section{Correlation b/w Proposed \& Existed TDS}

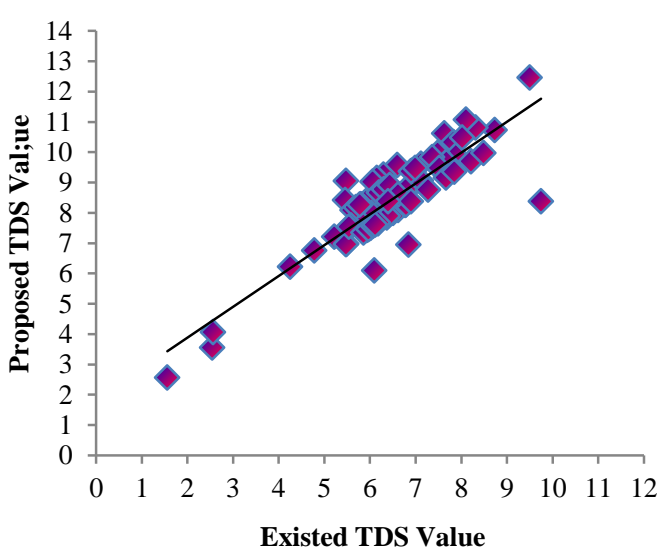

Fig. 7. Correlation b/w Proposed and Existed TDS 


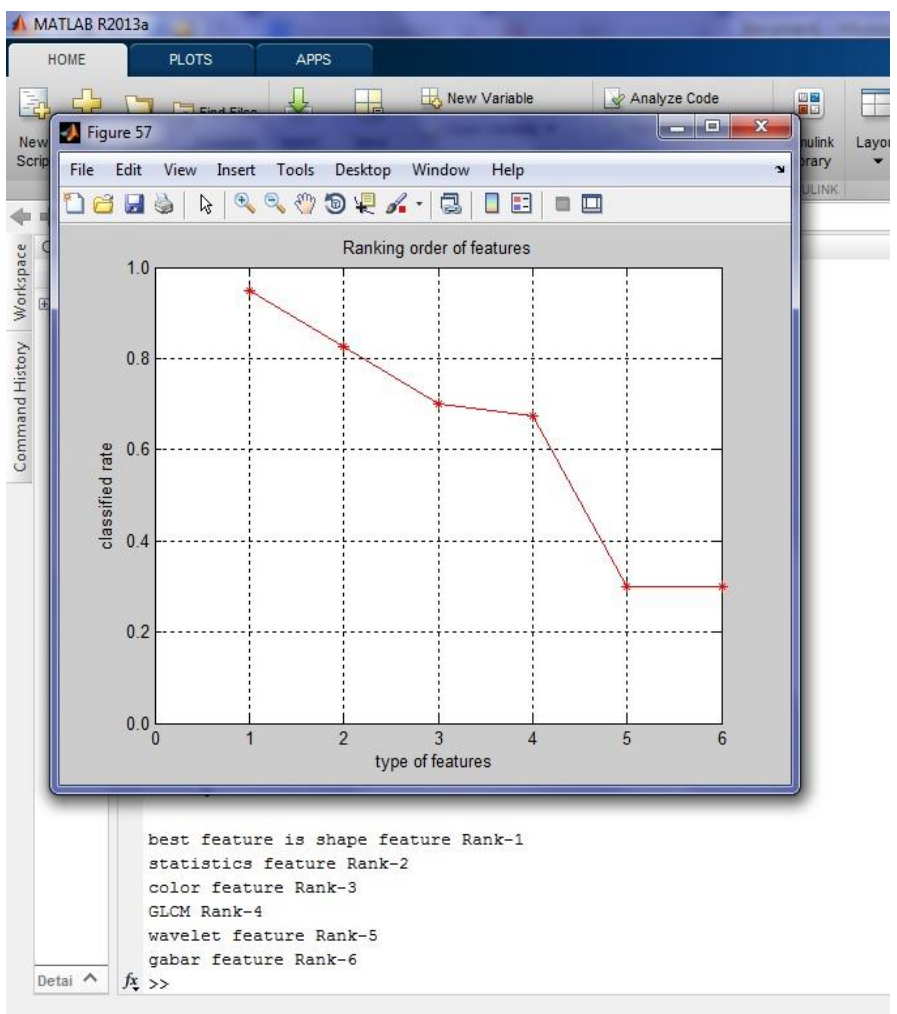

Fig. 8. Positing the Order of Features.

TABLE III. Estimations of True Positives/Negatives

\begin{tabular}{|l|l|l|}
\hline & Infected $(+)$ & Not Infected (-) \\
\hline Result Positive & True Positive $=75$ & False Positive=1 \\
\hline Result Negative & False Negative=3 & True Negative=3 \\
\hline
\end{tabular}

\section{CONCLUSION}

Early melanoma skin cancer diagnostic system is more efficient using a proposed TDS where the sensitivity and accuracy is improved compared to other conventional methods. The cost and time taken to detect is lower in this proposed methodology. Shape feature are determined as the most prominent feature for detecting the melanoma at early stage.

\section{REFERENCES}

[1] H. Ganster et al. Automated Melanoma Recognition. IEEE Transactions on Medical Imaging.2001, Vol. 20:3, pp. 233 - 239.

[2] T. Tanaka, R. Yamada, M. Tanaka, K. Shimizu, M. Tanaka. A Study on the Image Diagnosis of Melanoma. IEEE Trans. on Image Processing. 2004 June, pp. 1010-1024.
[3] T. Wadhawan, N. Situ, K. Lancaster, X. Yuan, G. Zouridakis. A portable library for melanoma detection on handheld devices in Biomedical Imaging. IEEE International Symposium. 2011, pp. 133136.

[4] T. Mendonca, P. M. Ferreira, J. S. Marques, A. R. Marcal, J. Rozeira. A dermoscopic image database for research and benchmarking in Engineering in Medicine and Biology Society (EMBC). 35th Annual International Conference of the IEEE. 2013, pp. 5437-5440.

[5] Pankaj Agrawal, S.K.Shriwastava , S.S.Limaye. MATLAB Implementation of Image Segmentation Algorithms. IEEE Pacific. pp.68-73.

[6] M. E. Celebi, Q. Wen, S. Hwang, H. Iyatomi, G. Schaefer. Lesion border detection in dermoscopy images using ensembles of thresholding methods. Skin Res. Technol. 2013 Feb ,19(1), pp. 252- 258.

[7] Mariam A.Sheha, MaiS.Mabrouk, AmrSharawy. Automatic Detection of Melanoma Skin Cancer using Texture Analysis. International Journal of Computer Applications. 2012, Volume 42.

[8] M. Sadeghi et al. A novel method for detection of pigment network in dermoscopic images using graphs. Computerized Medical Imaging and Graphics. 2010.

[9] G. Argenziano, G. Fabbrocini, P. Carli, V. de Giorgi, E. Sammarco, M. Delfino. Epiluminescence microscopy for the diagnosis of doubtful melanocytic skin lesions. Comparison of the ABCD rule of dermatoscopy and a new 7-point checklist based on pattern analysis. Arch. Dermatol. 1998 Dec , 134(12), pp. 1563-1570.

[10] Bilqis Amaliah1, Chastine Fatichah1, M. Rahmat Widyanto2. ABCD FEATURE EXTRACTION OF IMAGE DERMATOSCOPIC BASED ON MORPHOLOGY ANALYSIS FOR MELANOMA SKIN CANCER DIAGNOSIS. 90Jurnal Ilmu Komputer dan Informasi . 2010 Juni ,Vol 3(2).

[11] Alvarez, A., Brown, F. M., Grzymala-Busse, J. W., Hippe, Z. S. Optimization of the ABCD formula used for melanoma diagnosis. Int. Conf. On Intelligent Information Processing and WEB Mining Systems. 2003June, pp. 233-240.

[12] C. C. Chan , J. W. Grzymala-Busse. Rough-set boundaries as a tool for learning rules from examples. Int. Symp. on Methodologies for Intelligent Systems.pp. 281-288.

[13] A. FidalgoBarata, E. Celebi, , J. Marques.Improving Dermoscopy Image Classification Using Color Constancy. IEEE Journal of Biomedical and Health Informatics. 2014.

[14] C. Barata, M. Ruela, M. Francisco, T. Mendonca, J. S. Marques. Two Systems for the Detection of Melanomas in Dermoscopy Images Using Texture and Color Features. IEEE. 2013,vol. 99, pp. 1- 15.

[15] Garnavi, M. Aldeen, J. Bailey. Computer-aided diagnosis of melanoma using border-and wavelet-based texture analysis. Information Technology in Biomedicine. IEEE Transactions. 2012,vol16, pp. 12391252.

[16] L. Swets , J. Weng. Using Discriminant Eigenfeatures for Image Retrieval. IEEE Transactions on Pattern Analysis and Machine Intelligence. 1996,18(8):831-836.

[17] Zhao, et al. Subspace Linear Discriminant Analysis for Face Recognition. Tech. Rep. CAR-TR-914. 1999.

[18] MM Mukaka. A guide to appropriate use of Correlation coefficient in medical research. Malawi Med J. 2012 Sep, v.24(3),PMC3576830.

[19] R. F. Bartlett. Linear Modelling of Pearson's product moment correlation coefficient: An application of Fisher's z-transformation. Journal of the Royal Statistical Society. Series D (The Statistician). 1993.Vol. 42, No. 1, pp. 45-53. 